\title{
Erratum to: The Role of Calcium in Ameliorating the Oxidative Stress of Fluoride in Rats
}

\author{
N. E. Mohamed ${ }^{1}$
}

Published online: 2 September 2015

(C) Springer Science+Business Media New York 2015

Erratum to: Biol Trace Elem Res (2015)

DOI 10.1007/s12011-015-0421-5

The author wishes to indicate the following correction to the referenced article. Under the heading "Biochemical and Hormonal Studies", in the second paragraph, the sentence "Serum levels of total thyroxine (T4) (Art. no. IM1447), total triiodothyronine (T3) (Art. no.IM1699-IM3287), and estradiol (E2) were estimated by radioimmunoassay (RIA) technique using solid phase coated tubes and tracer labeled with ${ }^{125} \mathrm{I}$ (purchased from Immunotech, Beckman Coulter Company, USA). Serum parathormone hormone was determined by chemiluminescence technique according to [27]" is incorrect.

It should have read:

Serum levels of total thyroxine (T4)(Art, No. IM1447), total triiodothyronine (T3)(Art, No. IM1699-IM3287) and estradiol (E2)(Art, No. A21854) were estimated using radioimmunoassay (RIA) technique using solid phase coated tubes and tracer was labeled with $\mathrm{I}^{125}$ purchased from Immunotech, A Beckman Coulter Company (Radiova 1,102 27 Prague 10, Czech Rupublic). Serum parathermone hormone was determined using chemiluminescence technique according to [27].

The online version of the original article can be found at http://dx.doi.org/ 10.1007/s12011-015-0421-5.

N. E. Mohamed

nelshahat1@gmail.com; naglaa22000@yahoo.com

1 Biological Applications Department, Nuclear Research Centre, Atomic Energy Authority, P.O. 13759, Qalyoubiah, Egypt 